\title{
Environmental Impacts of China's Urbanization from 2000 to 2010 and Management Implications
}

\author{
Yu Tao ${ }^{1} \cdot{\text { Feng } \mathrm{Li}^{1} \cdot \text { John C. Crittenden }}^{2}$. \\ Zhongming $\mathrm{Lu}^{2} \cdot$ Xiao Sun ${ }^{1}$
}

Received: 26 March 2015/Accepted: 16 September 2015/Published online: 24 September 2015

(C) Springer Science+Business Media New York 2015

\begin{abstract}
Rapid urbanization in China during the first decade of the twenty first century has brought about profound environmental changes at citywide and regional scales. In this paper, we present a comprehensive set of indicators and put forward a new evaluation method for measuring environmental impacts of urbanization from 2000 to 2010. We compared these impacts among 286 cities in mainland China and found that the overall quantity of pollutant discharge decreased as cities became more economically developed during the years 2000-2010. However, larger and denser cities, and wealthier cities in the eastern part of China tended to have larger quantities of pollutant discharge, resource consumption, and changes in
\end{abstract}

Electronic supplementary material The online version of this article (doi:10.1007/s00267-015-0614-x) contains supplementary material, which is available to authorized users.

Feng Li

lifeng@rcees.ac.cn

Yu Tao

taoyu880216@126.com

John C. Crittenden

john.crittenden@ce.gatech.edu

Zhongming Lu

Zhongming.Lu@gatech.edu

Xiao Sun

sxdreamfree@163.com

1 State Key Laboratory of Urban and Regional Ecology, Research Center for Eco-Environmental Sciences, Chinese Academy of Sciences, 18 Shuangqing Road, Haidian District, Beijing 100085, China

2 Brook Byers Institute for Sustainable Systems, School of Civil \& Environmental Engineering, Georgia Institute of Technology, 828 West Peachtree St. NW, Suite 320, Atlanta, GA 30332-0595, USA land use (i.e., expansion of the built environment). The discharge increase occurred despite these cities having increased their investment in pollution control and construction of municipal environmental infrastructure. The negative impact from the intensity of pollutant discharge (i.e., discharge per unit of economic output) was generally less in more developed cities, although this was not always the case. Some cities, such as resource-based cities and old industrial cities, had both larger quantities of pollutant discharge and greater pollution intensity compared to other types of cities, indicating that environmental impacts did not necessarily decrease with increasing urbanization. The results of this study provide a promising basis for decisionmaking to reduce the impacts for different types of cities in the decades to come.

Keywords Environmental impacts - Urbanization · Comparative study $\cdot$ Megacities $\cdot$ Sustainable cities

\section{Introduction}

With rapid economic development and expansion of the built environment, urbanization has taken place at an unprecedented rate in China since the year 2000. The proportion of the Chinese population living in urban areas reached $50 \%$ in 2010 , whereas it was $36 \%$ only a decade previously. This trend is expected to continue, with a projected increase of 270 million in China's urban population over the next two decades (United Nations 2010). The urban built-up land area in China has grown by $78.5 \%$ over the past decade-even faster than its urban population, which grew by $46 \%$ (Bai et al. 2014). This rapid urbanization has resulted in many aspects of environmental change, and pollutant discharges, resource consumption, 
and changes in land use have affected large areas. A large body of literature has been published on urbanization and its impact on urban expansion, agricultural land use, net primary productivity, and rural transformation development (Long et al. 2011; Lu et al. 2011; Xu et al. 2011; Xu and Min 2013; Tian and Qiao 2014). Several other studies have also evaluated impacts of China's urbanization from various aspects of carbon dioxide emissions, air pollution, health effects, and sustainability dilemmas (Minx et al. 2011; Zhu et al. 2011; Bai et al. 2012; Lamsal et al. 2013).

Quantitative methodologies for modeling the impact of urbanization also have been developed in these literatures. For example, urban metabolism and material/energy flows analysis are widely used in studies focusing on urban energy consumption and emissions reduction in China $\mathrm{Su}$ et al. 2012; Feng et al. 2013; Chen et al. 2014; Chen and Chen 2015). Evaluation indicator system approaches are another big part of the methodologies. Although several studies have quantified environmental impacts based on similar indicator system ( $\mathrm{Li}$ et al. 2009; Liu et al. 2011; Li et al. 2012), the adoption of suitable indicators and reliable methods still requires attention to provide a basis for a more accurate understanding of environmental effects from urbanization. Moreover, studies that have integrated the sustainable development approach to modeling environmental effects from urbanization with investigation of the coupling model of coordination between urbanization and the environment have generally focused on individual cities or regions (Liu et al. 2011; Li et al. 2012).

In summary, recent research has either covered one or two aspects of environmental impacts over a short period of time, or has included only a few cities in some parts of China. No research has comprehensively evaluated these impacts on a broad scale during the first decade of the twenty first century or has compared the impacts among numerous cities across China. However, these extremely urgent issues need to be addressed. Our aim is to develop a comprehensive set of indicators and put forward a new evaluation method for measurement of environmental impacts of urbanization from 2000 to 2010 . In doing this, we compare the results across 286 cities in mainland China. Our findings provide a promising basis for decisionmaking that may reduce the impacts for different types of cities in the decades to come.

\section{Materials and Methods}

\section{Study Area and Classification of the Cities}

We classified and compared 286 cities in mainland China (not including Taiwan, Hong Kong, Macao, and Tibet) and their environmental impacts using criteria from the central government. Cities with different population sizes were defined as follows: megacities (above 3 million), large cities (between 1 and 3 million), medium-sized cities (between 500,000 and 1 million), and small cities (below 500,000) (China Society of Urban Economy 2010; Zhou et al. 2013). Cities with different geographic locations were divided hierarchically into three regions (east China, central China, and west China), seven subregions (east China, central China, north China, northeast China, northwest China, south China, and southwest China), and six urban agglomerations based on current administrative, geographic, and economic divisions in China (Fig. 1). Cities with different levels of environmental investment were classified into four types based on their honorary titles awarded from the central government (e.g., the Ministry of Environmental Protection of China) for their larger investments in (1) environmental protection, (2) ecological progress, and (3) both attributes, as compared to (4) cities with none of these attributes. We also examined cities based on the following classifications set by the government: (1) resource-based cities (i.e., those whose economic growth was sustained by exploitation of natural resources such as fossil fuels, mineral resources, or rare earth elements), (2) old industrial cities, (3) riverside and coastal cities, (4) northern cities, and (5) southern cities. These government classifications of cities were useful in exploring the variation in environmental impacts of urbanization in cities with different resource endowments, industrial structures, and geographic locations.

\section{Measurement Indicators and Data Sources}

Measurement indicators were grouped into two categories-urbanization and environmental impacts-and a total of 11 indicators were developed for each category (Fig. 2a, b). These indicators characterized urbanization based on urban size (GDP, built-up area, and total population), urban economy (per capita income for urban residents, per capita local financial revenue, and per capita industrial output), urban land area (per capita industrial area, per capita residential area, and per capita road area), and urban population (population density and urban population). The indicators characterized environmental impacts based on environmental pollution (industrial exhaust gas emissions, urban sewage discharge, and domestic garbage discharge), pollution intensity (exhaust gas emissions per 10,000 RMB of industrial output, urban sewage discharge per 10,000 RMB of GDP, and per capita domestic garbage discharge), land use change (per capita urban green space area, per capita arable land area, and per capita peri-urban open space area that included unused land such as woodland, shrubland, grassland, wetland, and water area), and resource consumption (water and energy 
Fig. 1 Spatial distribution of Chinese cities and their variation in population size in 2010

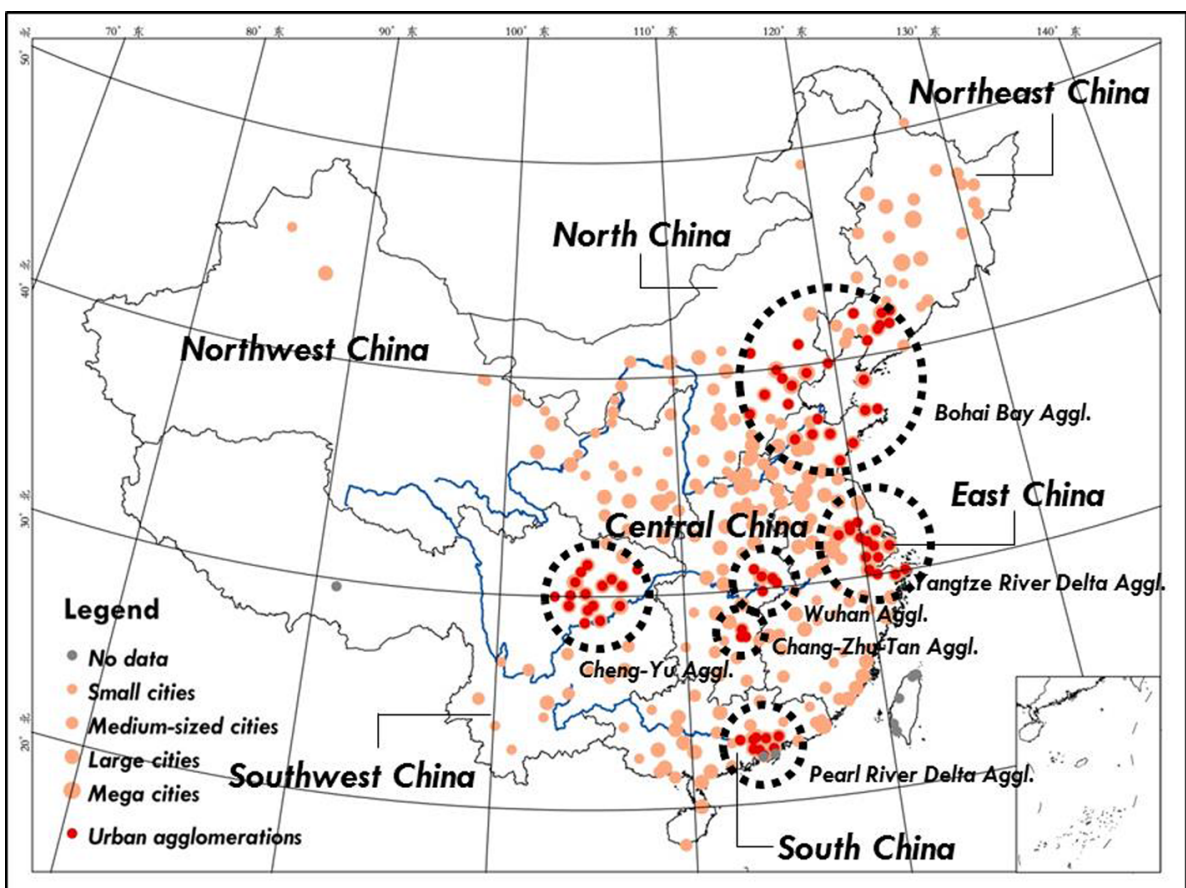

consumption per 10,000 RMB of GDP). The data were collected from the China City Statistical Yearbook, the China Urban Construction Statistical Yearbook, and the China Statistical Yearbook for Regional Economy (Ministry of Housing and Urban-Rural Development of China 2000-2010; National Bureau of Statistics of China 2001-2011a; National Bureau of Statistics of China 2001-2011b). The scope of the data covered urban areas exclusively, except for indicators of industrial exhaust gas emissions (including total emissions and emissions per unit of economic output), per capita arable land area, and per capita peri-urban open space area, which covered both urban and peri-urban areas.

\section{Data Standardization and Indicator Synthesis}

We used the Full Permutation Polygon Synthetic Indicator method for data standardization and indicator synthesis ( $\mathrm{Li}$ et al. 2009). For this method, an $n$-sided polygon is created to represent each of $n$ indicators. The distance from the center of each vertex (the distance from the center of the polygon) represents the magnitude of the indicators. Thus, there are $(n-1) ! / 2 n$-sided polygons. The theoretically largest value is the farthest from the center of the polygon and is normalized to have a value set to 1 . The center of the polygon has a value of -1 . The synthetic indicator indicates a comprehensive performance on all the measurement indicators, and is defined as the mean of the ratios of the area of any $n$-sided polygon to the area of the polygon defined using a normalized value of 1 for each indicator.
The standardization equation is as follows:

$S_{i}=\frac{\left(U_{i}-L_{i}\right)\left(X_{i}-T_{i}\right)}{\left(U_{i}+L_{i}-2 T_{i}\right) X_{i}+U_{i} T_{i}+L_{i} T_{i}-2 U_{i} L_{i}}$,

where $U_{i}, L_{i}$, and $T_{i}$ represent the upper limit, lower limit, and threshold, respectively, for parameter $X_{i}$. $S_{i}$ is the standardized value that ranges between $[-1,+1]$ and meets the following conditions: when $X_{i}=L_{i}, S_{i}=-1$; when $X_{i}=T_{i}, S_{i}=0$; and when $X_{i}=U_{i}, S_{i}=1$.

The value of the synthetic indicator is calculated as

$S=\frac{\sum_{i \neq j}^{i, j}\left(S_{i}+1\right)\left(S_{j}+1\right)}{2 n(n-1)}$,

where $S$ is the value of the synthetic indicator that ranges between $[0,1]$. Each of the indicators used for calculating the synthetic indicator are equally weighted.

Our method has the following two advantages. First, the standardization equation rescales the value of each indicator from $\left[L_{i}, U_{i}\right]$ to $[-1,+1]$. The original value of an indicator increases linearly from $L_{i}$ to $U_{i}$, while the standardized value of that indicator increases nonlinearly from -1 to 1 . The standardized value increases slowly if the value of the indicator is below the threshold and increases rapidly if the value is above the threshold (Fig. S1, See Appendix A. for Supplementary data). Second, the synthetic method alters the traditional additive approach to combine indicators using a multidimensional approach that better reflects the integrative system principle that the whole can be more than the sum of its parts. The value of the synthetic indicator is minimized if the values of the 

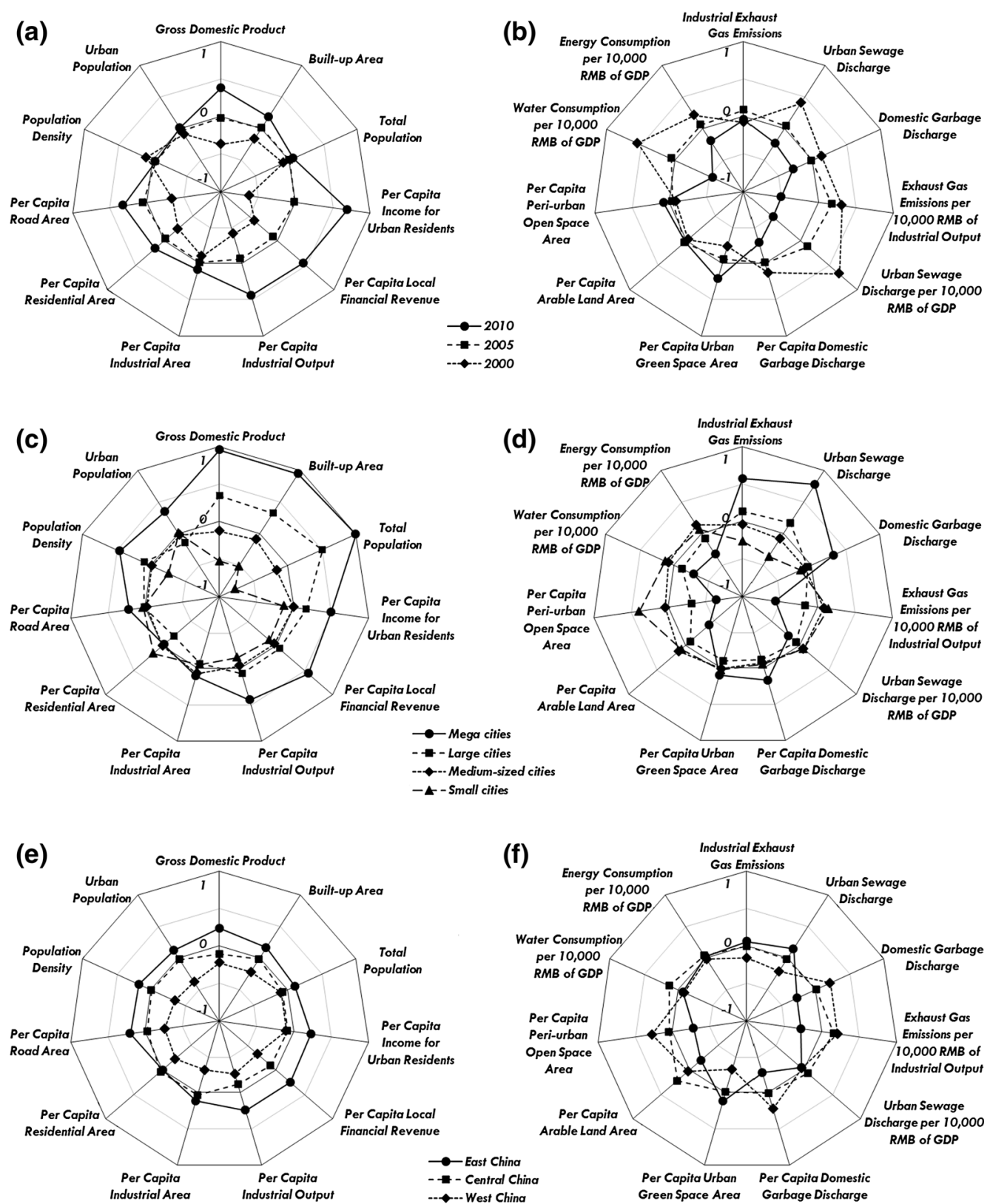

(g)

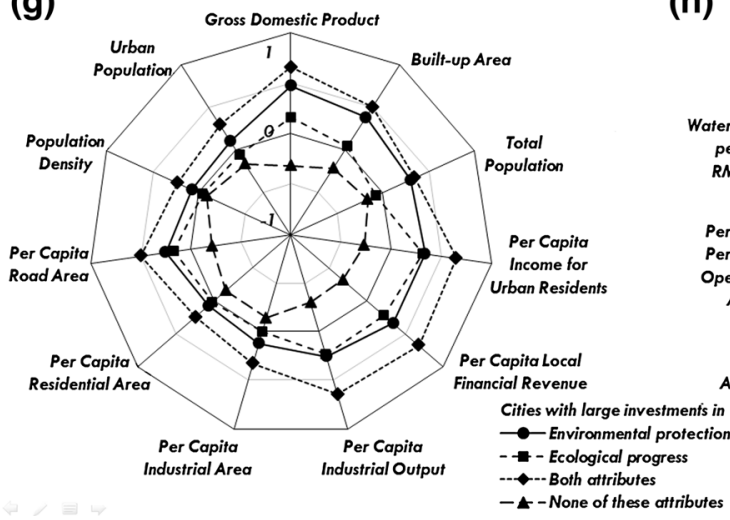

(h)
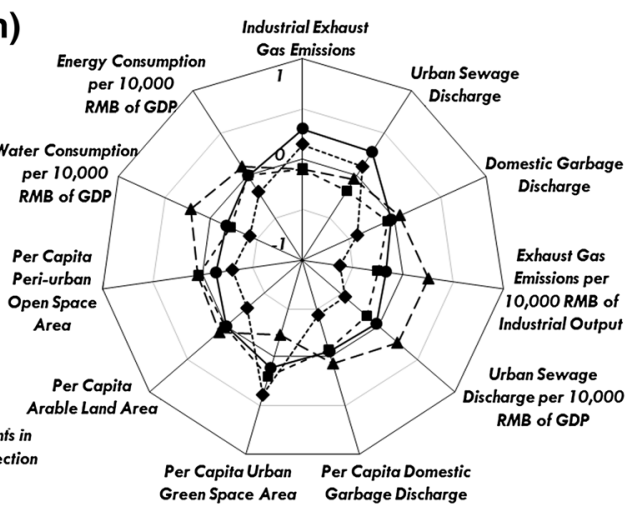
4Fig. 2 Values of the indicators for urbanization and environmental impacts (a, b) in the years 2000, 2005, and 2010; $\mathbf{c}$ and $\mathbf{d}$ in cities with different population sizes; $\mathbf{e}$ and $\mathbf{f}$ in cities with different geographic locations; and $\mathbf{g}, \mathbf{h}$ in cities with different levels of environmental investment

indicators are below the thresholds and the side lengths are less than 1 and is magnified if the values are above the thresholds and the side lengths are greater than 1 . These advantages enable sensitive identification of cities with upper and lower values for each indicator, and visibly highlight hot spots and cold spots.

To calculate the standardized value, upper and lower limits and thresholds are required. We determined these by first sorting a total of 858 cities for the years 2000, 2005, and 2010 according to their indicator values in descending order. Next, we used values of the upper and lower 5th percentile as the upper and lower limits, and used median value as the threshold for each indicator (Table S1, See Appendix A. for Supplementary data). We then set the standardized values of cities in the upper and lower 5th percentile at 1 and -1 , respectively. This eliminated negative impact of maximum and minimum values of the indicator on data standardization. The standardized values were evenly distributed within the range of $[-1,+1]$. If the standardized value of an indicator for a city was positive, it indicated the indicator value of the city was in the front rank, and vice versa.

In calculating the value of the synthetic indicator, the indicators can either be equally weighted or unequally weighted (Jin et al. 2011). It was unreasonable to set the same weight for indicators for different types of cities but set different weights for different indicators. However, setting different weights for indicators for each type of city brought about great uncertainties and results that were not comparable. Therefore, the indicators were all equally weighted in this research.

We calculated the synthetic indicator for "Urbanization" by including all 11 indicators as shown in Fig. 2a. We also calculated two synthetic indicators for environmental impacts as shown in Fig. 2b. One synthetic indicator was "Pollution Intensity and Resource Consumption", which indicated the intensity of impacts per unit of economic output, including exhaust gas emissions per 10,000 RMB of industrial output, urban sewage discharge per 10,000 RMB of GDP, per capita domestic garbage discharge, and water and energy consumption per 10,000 RMB of GDP. The other synthetic indicator was "Environmental Pollution and Land Use Change", which indicated the total quantity of impacts, including industrial exhaust gas emissions, urban sewage discharge, domestic garbage discharge, per capita arable land area, and per capita peri-urban open space area.

We did not include per capita urban green space area in calculating the synthetic indicator because its variation across different city types was counter to that of the above five indicators. We found that if one city type had a greater value of per capita urban green space area (indicating lower impacts), it could possibly have smaller values of per capita arable land area and per capita peri-urban open space area and greater values of industrial exhaust gas emissions, urban sewage discharge, and domestic garbage discharge (indicating greater impacts in both cases) as compared to another type of city, and vice versa. The exclusion of per capita urban green space area, therefore, improved our ability to identify variation of the synthetic indicator across different city types. In addition, since lower values of per capita arable land area and per capita peri-urban open space area resulted in greater impacts, as shown in Fig. 2, we exchanged their upper limits with lower limits in the standardization process of these two indicators. In this way, lower original values resulted in greater standardized values and hence a greater value of the synthetic indicator for Environmental Pollution and Land Use Change, as shown in Fig. 3.

\section{Results}

\section{Environmental Impacts of Urbanization in Cities from 2000 to 2010}

The synthetic indicator for urbanization for 286 Chinese cities increased from 0.17 to 0.42 during the years 2000-2010, with expansion in urban size in terms of economic, spatial, and demographic aggregations (Fig. 3a). Economic growth for local government and industrial sectors tripled during that period, as did per capita income for urban residents. The expansion of the built-up area that included residential land, industrial land, and land for roads almost doubled. Population structure (i.e., the proportion of urban population to total population) and size grew slowly but steadily (Fig. 2a).

The synthetic indicator for environmental pollution and land use change decreased from 0.30 to 0.18 , while the synthetic indicator for pollution intensity and resource consumption showed a greater decrease, from 0.47 in 2000 to 0.10 in 2010 (Fig. 3a). Urban sewage discharge and domestic garbage discharge totals in 286 Chinese cities decreased slightly during this period; however, industrial exhaust gas emissions (including $\mathrm{SO}_{2}$ and soot emissions) remained high. In contrast, the intensity of pollutant discharge and of water and energy (electricity) consumption per unit of economic output decreased by one-third to twothirds over these 10 years, primarily because of the rapid growth in GDP and industrial output. At the same time, per capita urban green space area increased by about $60 \%$, demonstrating positive results of China's efforts to increase 

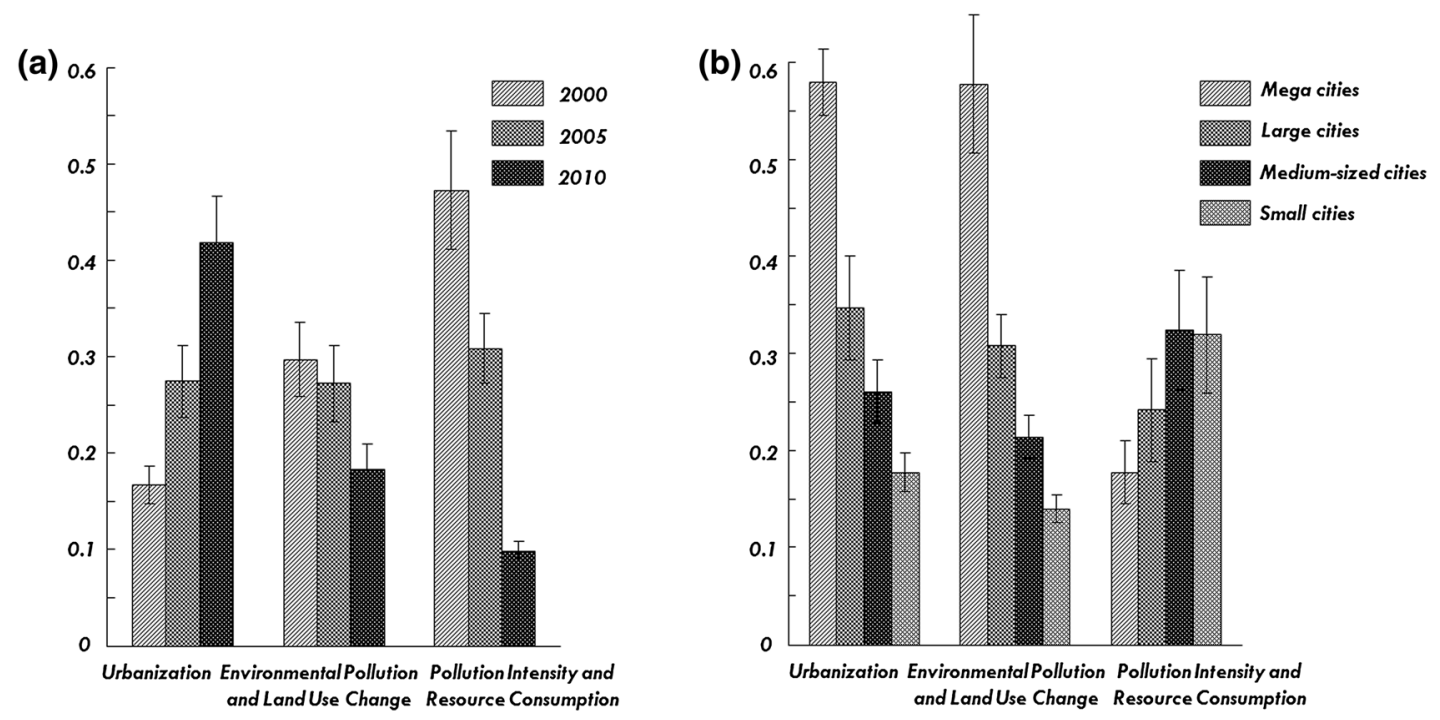

(c)

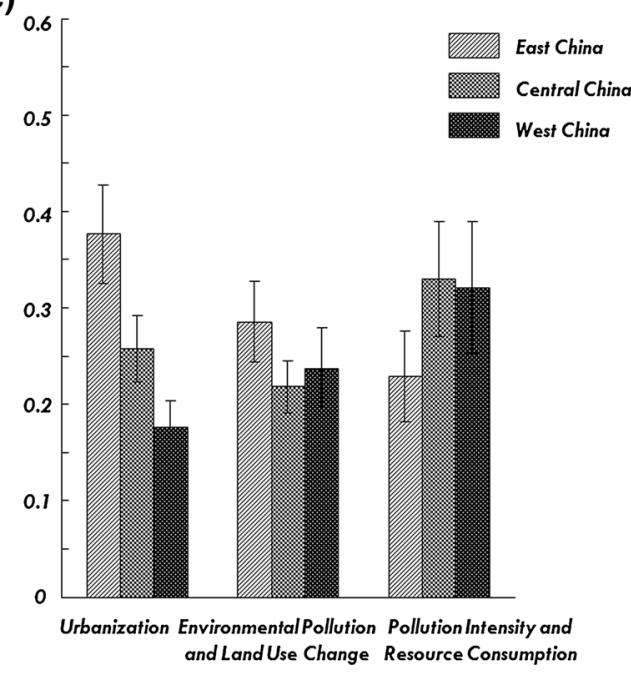

Fig. 3 Values of the synthetic indicators for urbanization and environmental impacts $\mathbf{a}$ in the years 2000, 2005, and 2010 ( $n=286$ for each year); $\mathbf{b}$ in megacities $(n=47)$, large cities $(n=281)$, medium-sized cities $(n=321)$, and small cities $(n=185)$; $\mathbf{c}$ in east China $(n=345)$, central China $(n=330)$, and

urban green space (Fuller and Gaston 2009; Chen and Wang 2013a, b; Zhao et al. 2013), while nationwide per capita arable land area and per capita peri-urban open space area remained stable. Consequently, the overall expansion of the built-up area caused low impacts on regional land use and covered only a small proportion of the total land area (Fig. 2b).

\section{Environmental Impacts of Urbanization in Cities with Different Population Sizes}

The indicator values for urban size, urban economy, urban land area, and urban population in megacities and large

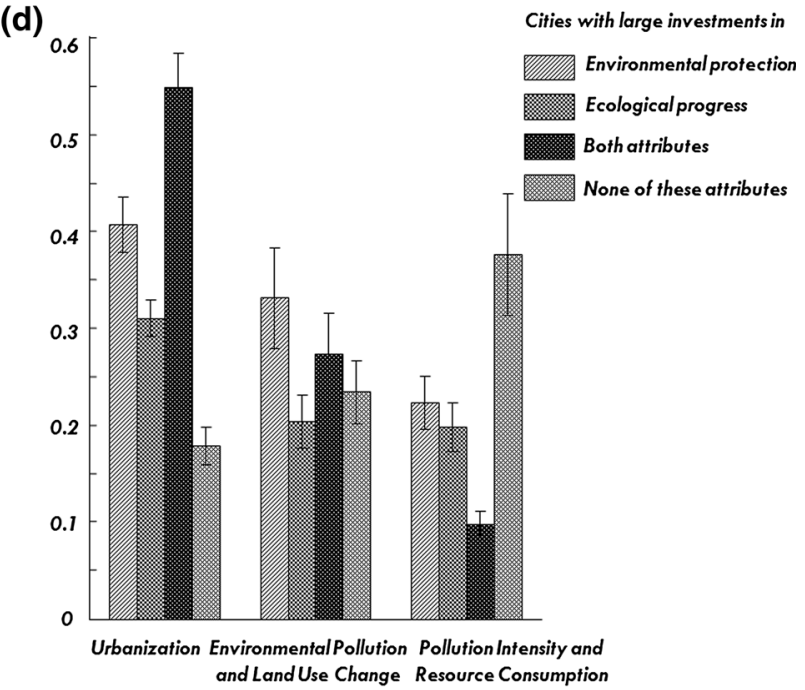

west China ( $n=183)$; and $\mathbf{d}$ in cities with larger investments in environmental protection $(n=84)$, ecological progress $(n=83)$, and both attributes $(n=168)$ as compared to cities with none of these attributes $(n=523)$ Error bars represent mean square deviation of the values within each type of city

cities were greater than those of small and medium-sized cities (Fig. 2c). The synthetic indicator for urbanization averaged 0.58 for megacities and 0.35 for large cities, both of which were greater than the 0.26 of medium-sized cities and 0.18 of small cities (Fig. 3b). In contrast, exhaust gas emissions per 10,000 RMB of industrial output were ranged from 20 to $60 \%$ less and urban sewage discharge per 10,000 RMB of GDP was from 10 to $20 \%$ less in megacities and large cities than those of small and medium-sized cities because GDP and industrial output were greater in megacities and large cities than those of small and medium-sized cities. Water and energy consumption per 10,000 RMB of GDP were ranged from 20 to $40 \%$ less 
in megacities and large cities than those of small and medium-sized cities, although the total quantity of resource consumption was much greater in megacities and large cities than that of small and medium-sized cities. Industrial exhaust gas emissions, urban sewage discharge, and domestic garbage discharge totals in megacities and large cities were 1.3-1.9, 1.5-2.3, and 1.1-1.5 times greater, respectively, than discharges from small and medium-sized cities, and this increased the severity of air, water, and soil pollution in the larger cities. Per capita urban green space area was greater in megacities and large cities, while per capita arable land area and per capita peri-urban open space area were from 50 to $80 \%$ less and 30 to $60 \%$ less, respectively, than those of small and medium-sized cities. This decrease can be attributed to the conversion of arable land and open space to the built environment-a land use change that was 1.5-4.0 times greater in megacities and large cities (Fig. 2d).

Although the synthetic indicator for pollution intensity and resource consumption was lower in megacities and large cities than in small and medium-sized cities, the synthetic indicator for environmental pollution and land use change of megacities was approximately twice that of large cities, three times that of medium-sized cities, and four times that of small cities (Fig. 3b). Accordingly, the overall environmental impacts were greater in megacities and large cities.

\section{Environmental Impacts of Urbanization in Cities with Different Geographic Locations}

The synthetic indicator for urbanization averaged 0.38 , 0.26 , and 0.18 in east, central, and west China, respectively (Figs. 2e, 3c). In terms of subregions, east China's average GDP was 0.16 (standardized between -1 and 1) and ranked first among the seven subregions, while south China had the greatest average per capita income for urban residents (0.25). Per capita road area averaged 0.20 in north China and 0.18 in east China, values that were greater than those of the other subregions. Per capita industrial area was greatest in northeast China (averaging 0.26) because it is the most important historical industrial base in China. Population density averaged 0.25 in central China, and this became one of the major driving factors of urbanization in this subregion. Southwest China and northwest China were less developed, with indicator values much lower than those of the other subregions. Urbanization indicator values in cities of the six urban agglomerations were 1.4-1.6 times greater than those of the other cities. The three largest urban agglomerations are in the Yangtze River Delta, the Pearl River Delta, and around the Bohai Bay. These regions had greater GDP, built-up area, and population size compared to the other urban agglomerations.

Industrial exhaust gas emissions and urban sewage discharge were greater in east China, while domestic garbage discharge to the environment without proper treatment was less for east China than for central and west China. This is because the standardized value of domestic garbage treatment ratio (percent of domestic garbage that is treated) averaged 0.30 in east China, only 0.02 in central China, and -0.24 in west China. Per capita urban green space area was greater in east China, while per capita arable land area was from 20 to $30 \%$ less and per capita peri-urban open space area was from 30 to $40 \%$ less than that of central and west China (Figs. 2f, 3c). As for comparisons between northern and southern cities, cities in north China had greater domestic garbage discharge, industrial exhaust gas emissions, and energy consumption totals, while urban sewage discharge and water consumption totals were greater in south China. Urban sewage discharge totals in riverside and coastal cities were about $40 \%$ greater than that of inland cities. Such extensive pollution would have a large impact on water quality, although the carrying capacity of the water environment was greater in riverside and coastal cities.

Among the seven subregions, the total discharge volume of industrial exhaust gas, urban sewage, and domestic garbage was relatively high in northeast China compared to the other subregions. Water pollution and water consumption were high in central and south China. Domestic garbage discharge in cities of the Cheng-Yu urban agglomeration in southwest China was about $70 \%$ greater than for the other cities (excluding cities of the other five urban agglomerations), and was about $60 \%$ greater in per capita terms. The total emissions of industrial exhaust gas as well as emissions per 10,000 RMB of industrial output in north China were about 50 and $20 \%$ greater, respectively, than for the other subregions. These emission levels were probably the most important factor causing heavy particulate matter pollution in this area (Ji et al. 2012; An et al. 2013; Cheng et al. 2013). Per capita urban green space area was relatively low in northwest China. The expansion of the built-up area in east China was evident by reduced area of per capita peri-urban open space, which was about $40 \%$ below the national average. Furthermore, per capita peri-urban open space area in cities of the Yangtze River Delta urban agglomeration in east China was about $80 \%$ less than that of the other cities, indicating that urban agglomeration resulted in greatly decreased rural areas. This posed potential impact on provision of ecosystem services at a regional scale (Liu et al. 2010; Wu et al. 2014; Xu et al. 2014). 


\section{Environmental Impacts of Urbanization in Cities with Different Levels of Environmental Investment}

As shown in Figs. 2g and 3d, cities with large investments in both environmental protection and ecological progress had the greatest urbanization indicator (averaging 0.55), followed by those with large investments in either environmental protection (averaging 0.41 ) or ecological progress (averaging 0.31). Cities with neither of these attributes had the smallest urbanization indicator (averaging 0.18). Per capita incomes for urban residents, local government, and industrial sectors in cities with larger investments in environmental protection and ecological progress were also 1.8-2.5 times greater than those of other cities, indicating that more economically developed cities were more likely to increase investments in environmental protection and ecological progress.

The intensity of pollutant discharge and of water and energy consumption per unit of economic output were from 10 to $30 \%$ less in cities that had large investments in ecological progress, 30-40\% less in cities with large investments in environmental protection, and 40-70 \% less in cities with large investments in both attributes compared to the other cities. Although these three types of cities had lower domestic garbage discharge totals, industrial exhaust gas emissions and urban sewage discharge totals were from 30 to $40 \%$ greater than for the other cities. Per capita arable land area and per capita peri-urban open space area were about $30 \%$ less in cities with large investments in environmental protection, ecological progress, or both, although these cities had greater per capita urban green space area compared to the other cities (Figs. 2h, 3d).

Several reasons may explain these findings. Cities that invested in ecological progress or environmental protection had a greater GDP, a more extensive built-up area, and a larger population size, all of which contributed to greater environmental impacts and also influenced the measurement indicators for determining the effectiveness of a city's investments in environmental protection and ecological progress. Cities that invested in ecological progress or environmental protection placed more emphasis on reducing the intensity of pollutant discharge per unit of economic output rather than on controlling the total discharge of pollutants.

\section{Discussion and Conclusion}

\section{Limitations of the Study}

We recognize that there are three limitations to this study. First, the cities were classified into different types according to size, location, or investments in environmental sustainability, and analyzed on the basis of one of these types, but the results were influenced by a combination of these types (i.e., confounding variables). For example, the larger quantity of pollutant discharge in cities with larger investments in environmental protection and ecological progress was mainly because about $70 \%$ of these cities were megacities and large cities. In contrast, megacities and large cities accounted for less than $30 \%$ of cities with neither of these attributes.

Second, although the main evidence came from the cross-sectional (between-city) variation of the data, the time-series (within-city) dimension provided some additional information. The results showed that more economically developed cities tended to have larger quantities of pollutant discharge as compared to less developed cities; however, pollutant discharge totals were reduced somewhat as cities became more developed than they once were during the years 2000-2010. Third, a small bias resulted because of a few missing values for some indicators, although this bias did not alter the order of values when averaged for different types of cities.

Econometric modeling of both direct and indirect driving factors of environmental impacts will be targeted in future research (Jiang et al. 2012; Zhao et al. 2012; Jiang et al. 2013). It will also be necessary to quantify spatial and temporal variations of these impacts of urbanization across cities using spatial statistical analyses.

\section{Implications of the Results}

The results of the study show high environmental impacts during the process of rapid urbanization in China from 2000 to 2010. It is expected that the growth of economy, population, and urban built-up land area will still push the increase of environmental pressure in the near future (Liang et al. 2014). On the other hand, solutions to these environmental problems will depend on the progress of urbanization in the coming decades. In part this is because rapid urbanization provides an enormous opportunity to introduce sustainable technologies and practices on a national scale (Bai et al. 2014). Our study found that cities invested more in environmental protection as they became more economically developed. For example, finances for pollution control and construction of municipal environmental infrastructure in 286 Chinese cities increased by about 80 and $30 \%$, respectively, during the years 2000-2010, and ranged from 20 to $80 \%$ greater and 30 to $90 \%$ greater, respectively, in megacities and large cities compared to small and medium-sized cities. This is also because more developed cities tend to have less pollutant discharge and resource consumption per unit of economic output, although this is not always the case. Some cities (such as resource-based cities and old industrial cities in 
this study) not only had larger quantities of pollutant discharge, but also had from 20 to $40 \%$ greater intensity of pollutant discharge per unit of economic output compared to the other cities, indicating that environmental impacts did not necessarily decrease with increasing urbanization. Economic growth through high energy and resource consumption, followed by the implementation of environmental protection to reduce environmental pollution, does not lead to sustainable growth (He and Wang 2012; Elliott et al. 2013).

In general, China needs timely technology innovation to reduce emissions intensity and should further focus on restructuring its economy in order to continue urban growth while ensuring environmental protection (Liang et al. 2013). China's policies on urbanization should comprehensively consider a wide range of environmental impacts and compare these impacts among numerous cities in order to be more tailored and adaptive with local economic and environmental contexts. Our results suggest that larger and denser cities, and wealthier cities in the eastern part of China, where imbalance emerges between the economy and the environmental aspect, are capable of investing sufficient money in environmental protection and ecological progress in order to control industrial exhaust gas emissions, urban sewage discharge, and domestic garbage discharge totals and to conserve arable land and open space in peri-urban and rural areas. While small and medium-sized cities and those in less developed regions of China should concentrate more on decreasing their pollutant discharge and resource consumption per unit of economic output, and on increasing their urban green space area in order to achieve bigger improvements in environmental performances in their future development. On the whole, the incentive policy of central government and local governments is necessary to facilitate cross-city learning and realize eco-friendly development in future. This will help balance development of GDP and population growth, resource utilization, and environmental protection.

Acknowledgments This research was funded by the National Natural Science Foundation of China (No. 71273254). We also appreciate support from the Brook Byers Institute for Sustainable Systems and Hightower Chair and Georgia Research Alliance at Georgia Institute of Technology. We thank Dr. Denise Rennis and Dr. Deborah Rogers for assistance with an early version of this manuscript.

\section{References}

An XQ, Hou Q, Li N, Zhai SX (2013) Assessment of human exposure level to $\mathrm{PM}_{10}$ in China. Atmos Environ 70:376-386

Bai XM, Chen J, Shi PJ (2012) Landscape urbanization and economic growth in China: positive feedbacks and sustainability dilemmas. Environ Sci Technol 46:132-139
Bai XM, Shi PJ, Liu YS (2014) Realizing China's urban dream. Nature 509:158-160

Chen SQ, Chen B (2015) Urban energy consumption: different insights from energy flow analysis, input-output analysis and ecological network analysis. Appl Energy 138:99-107

Chen WY, Wang DT (2013a) Economic development and natural amenity: an econometric analysis of urban green spaces in China. Urban For Urban Green 12:435-442

Chen WY, Wang DT (2013b) Urban forest development in China: natural endowment or socioeconomic product? Cities 35:62-68

Chen SQ, Chen B, Fath BD (2014) Urban ecosystem modeling and global change: potential for rational urban management and emissions mitigation. Environ Pollut 190:139-149

Cheng Z, Jiang JK, Fajardo O, Wang SX, Hao JM (2013) Characteristics and health impacts of particulate matter pollution in China (2001-2011). Atmos Environ 65:186-194

Committee for Economic Development of Small and Medium-sized Cities of the China Society of Urban Economy (2010) Green book of small and medium-sized cities: annual report on development of small and medium-sized cities in China 2010. Social Sciences Academic Press, Beijing (in Chinese)

Elliott RJR, Sun PY, Chen SY (2013) Energy intensity and foreign direct investment: a Chinese city-level study. Energy Econ 40:484-494

Feng YY, Chen SQ, Zhang LX (2013) System dynamics modeling for urban energy consumption and $\mathrm{CO}_{2}$ emissions: a case study of Beijing, China. Ecol Modell 252:44-52

Fuller RA, Gaston KJ (2009) The scaling of green space coverage in European cities. Biol Lett 5:352-355

He J, Wang H (2012) Economic structure, development policy and environmental quality: an empirical analysis of environmental Kuznets curves with Chinese municipal data. Ecol Econ 76:49-59

Ji DS, Wang YS, Wang LL, Chen LF, Hu B, Tang GQ, Xin JY, Song T, Wen TX, Sun Y, Pan YP, Liu ZR (2012) Analysis of heavy pollution episodes in selected cities of northern China. Atmos Environ 50:338-348

Jiang L, Deng XZ, Seto KC (2012) Multi-level modeling of urban expansion and cultivated land conversion for urban hotspot counties in China. Landsc Urban Plann 108:131-139

Jiang L, Deng XZ, Seto KC (2013) The impact of urban expansion on agricultural land use intensity in China. Land Use Pol 35:33-39

Jin JS, Wang RS, Li F, Huang JL, Zhou CB, Zhang HT, Yang WR (2011) Conjugate ecological restoration approach with a case study in Mentougou district, Beijing. Ecol Complex 8:161-170

Lamsal LN, Martin RV, Parrish DD, Krotkov NA (2013) Scaling relationship for $\mathrm{NO}_{2}$ pollution and urban population size: a satellite perspective. Environ Sci Technol 47:7855-7861

Li F, Liu XS, Hu D, Wang RS, Yang WR, Li D, Zhao D (2009) Measurement indicators and an evaluation approach for assessing urban sustainable development: a case study for China's Jining City. Landsc Urban Plann 90:134-142

Li YF, Li Y, Zhou Y, Shi YL, Zhu XD (2012) Investigation of a coupling model of coordination between urbanization and the environment. J Environ Manage 98:127-133

Liang S, Xu M, Suh S, Tan RR (2013) Unintended environmental consequences and co-benefits of economic restructuring. Environ Sci Technol 47:12894-12902

Liang S, Liu Z, Crawford-Brown D, Wang YF, Xu M (2014) Decoupling analysis and socioeconomic drivers of environmental pressure in China. Environ Sci Technol 48:1103-1113

Liu YS, Wang JY, Long HL (2010) Analysis of arable land loss and its impact on rural sustainability in Southern Jiangsu Province of China. J Environ Manage 91:646-653

Liu YB, Yao CS, Wang GX, Bao SM (2011) An integrated sustainable development approach to modeling the eco-environmental effects from urbanization. Ecol Indic 11:1599-1608 
Long HL, Zou J, Pykett J, Li YR (2011) Analysis of rural transformation development in China since the turn of the new millennium. Appl Geogr 31:1094-1105

Lu QS, Liang FY, Bi XL, Duffy R, Zhao ZP (2011) Effects of urbanization and industrialization on agricultural land use in Shandong Peninsula of China. Ecol Indic 11:1710-1714

Ministry of Housing and Urban-Rural Development of China (2000-2010) China Urban Construction Statistical Yearbook 2000-2010. China Planning Press, Beijing (in Chinese)

Minx JC, Baiocchi G, Peters GP, Weber CL, Guan DB, Hubacek K (2011) A "carbonizing dragon": China's fast growing $\mathrm{CO}_{2}$ emissions revisited. Environ Sci Technol 45:9144-9153

National Bureau of Statistics of China (2001-2011a) China City Statistical Yearbook 2001-2011. China Statistics Press, Beijing (in Chinese)

National Bureau of Statistics of China (2001-2011b) China Statistical Yearbook for Regional Economy 2001-2011. China Statistics Press, Beijing (in Chinese)

Population Division, Department of Economic and Social Affairs, United Nations (2010) World urbanization prospects: the 2009 Revision. United Nations, New York

Su MR, Chen L, Chen B, Chen SQ, Yang ZF (2012) Low-carbon development patterns: observations of typical Chinese cities. Energies 5:291-304

Tian GJ, Qiao Z (2014) Assessing the impact of the urbanization process on net primary productivity in China in 1989-2000. Environ Pollut 184:320-326
Wu SH, Zhou SL, Chen DX, Wei ZQ, Dai L, Li XG (2014) Determining the contributions of urbanisation and climate change to NPP variations over the last decade in the Yangtze River Delta, China. Sci Total Environ 472:397-406

Xu XL, Min XB (2013) Quantifying spatiotemporal patterns of urban expansion in China using remote sensing data. Cities 35:104-113

Xu Y, Tang Q, Fan J, Bennett SJ, Li Y (2011) Assessing construction land potential and its spatial pattern in China. Landsc Urban Plann 103:207-216

Xu XB, Tan Y, Chen S, Yang GS (2014) Changing patterns and determinants of natural capital in the Yangtze River Delta of China 2000-2010. Sci Total Environ 466-467:326-337

Zhao JJ, Chen SB, Wang H, Ren Y, Du K, Xu WH, Zheng H, Jiang B (2012) Quantifying the impacts of socio-economic factors on air quality in Chinese cities from 2000 to 2009. Environ Pollut 167:148-154

Zhao JJ, Chen SB, Jiang B, Ren Y, Wang H, Vause J, Yu HD (2013) Temporal trend of green space coverage in China and its relationship with urbanization over the last two decades. Sci Total Environ 442:455-465

Zhou SY, Dai JC, Bu JH (2013) City size distributions in China 1949 to 2010 and the impacts of government policies. Cities 32:S51S57

Zhu YG, Ioannidis JPA, Li H, Jones KC, Martin FL (2011) Understanding and harnessing the health effects of rapid urbanization in China. Environ Sci Technol 45:5099-5104 